\title{
Toward a Consensus Model of the hERG Potassium Channel
}

\author{
Anna Stary, ${ }^{*[a, d]}$ Sören J. Wacker, ${ }^{[a]}$ Lars Boukharta, $^{[\mathrm{b}]}$ Ulrich Zachariae, ${ }^{[\mathrm{a}]}$ \\ Yasmin Karimi-Nejad, ${ }^{[\mathrm{c}]}$ Johan Åqvist, ${ }^{[\mathrm{b}]}$ Gert Vriend, $^{[\mathrm{d}]}$ and Bert L. de Groot ${ }^{[\mathrm{a}]}$
}

\begin{abstract}
Malfunction of hERG potassium channels, due to inherited mutations or inhibition by drugs, can cause long QT syndrome, which can lead to life-threatening arrhythmias. A three-dimensional structure of hERG is a prerequisite to understand the molecular basis of hERG malfunction. To achieve a consensus model, we carried out an extensive analysis of hERG models based on various alignments of helix S5. We analyzed seven models using a combination of conventional geometry/packing/normality validation methods as well as molecular dynamics simulations and molecular docking. A synthetic test set with the X-ray crystal structure of $\mathrm{K}_{\mathrm{v}} 1.2$ with artificially shifted S5 sequences modeled into the structure served as a reference
\end{abstract}

case. We docked the known hERG inhibitors (+)-cisapride, (S)terfenadine, and MK-499 into the hERG models and simulation snapshots. None of the single analyses unambiguously identified a preferred model, but the combination of all three revealed that there is only one model that fulfils all quality criteria. This model is confirmed by a recent mutation scanning experiment (P. Ju, G. Pages, R. P. Riek, P. C. Chen, A. M. Torres, P. S. Bansal, S. Kuyucak, P. W. Kuchel, J. I. Vandenberg, J. Biol. Chem. 2009, 284, 1000-1008). ${ }^{[1]}$ We expect the modeled structure to be useful as a basis both for computational studies of channel function and kinetics as well as the design of experiments.

\section{Introduction}

The human ether-à-go-go-related gene ( $h E R G$ ) encodes the pore-forming subunits of potassium channels that conduct the rapid delayed rectifier $\mathrm{K}^{+}$current $\left(I_{\mathrm{Kr}}\right)^{[2,3]} I_{\mathrm{Kr}}$ is activated by membrane depolarization and is a key determinant for re-polarization of the cell membrane during the cardiac action potential. ${ }^{[4,5]}$ hERG is up-regulated in various cancer cell lines, suggesting its role in the pathophysiology of cancer. ${ }^{[6]}$ Mutations in the hERG gene can cause inherited long QT syndrome (LQTS), a disorder that predisposes affected individuals to lifethreatening arrhythmias and sudden death. ${ }^{[7]}$ Blockade of hERG can lead to acquired LQTS, a rare side effect of treatment with structurally diverse medications. ${ }^{[8]}$ This potential for QT prolongation has led to severe restriction or withdrawal of several medications from the market. Intense efforts are directed at gaining a better understanding of the molecular basis of hERG channel blockade, including in vivo, in vitro, and in silico approaches (for a review, see reference [9]). Several groups have presented homology models of the hERG pore domain, providing a qualitative insight into potential ligand-channel interactions (for examples, see references [10-14]), and in some cases quantitative predictions have been provided. ${ }^{[15-17]}$

The accuracy of homology models depends critically on the sequence identity between template and target. ${ }^{[18,19]}$ The S5 helices of hERG are only distantly related to potential templates such as $\mathrm{KcsA}_{1}{ }^{[20]} \mathrm{MthK}_{1}{ }^{[21]} \mathrm{K}_{\mathrm{v}} \mathrm{AP}_{1}{ }^{[22]}$ or $\mathrm{K}_{\mathrm{v}} 1.2,{ }^{[23,24]}$ with sequence identities $<30 \%$. Consequently, no consensus has been established regarding the optimal alignment for S5. Differences in alignment of segment S5 have been neglected; however, S5 helices are in close contact with S6 segments, and are thus likely to influence the drug binding site. Therefore, we tested seven hERG models with different S5 alignments, five of which have been published, ${ }^{[11-14,16]}$ with a combination of state-of-the-art quality assessment methods and molecular dynamics (MD) simulations, and then analyzed the consequences of alignment errors on drug-receptor studies. To avoid potential errors and biases from quality assessment programs, a set of "control" structures, consisting of a native $\mathrm{K}_{\mathrm{v}} 1.2$ crystal structure, and "artificial" models, with shifted helices, was included in our study. Generally, a quality check must be able to verify the biological relevance of a model; that is, should be able to identify improperly folded models that result from alignment

[a] Dr. A. Stary, S. J. Wacker, Dr. U. Zachariae, Prof. Dr. B. L. de Groot Computational Biomolecular Dynamics Group Max Planck Institute for Biophysical Chemistry Am Fassberg 11, 37077 Göttingen (Germany)

Fax: (+49) 551-2012302 E-mail:anna.stary@univie.ac.at

[b] L. Boukharta, Prof. Dr. J. Åqvist Department of Cell and Molecular Biology Uppsala University, Biomedical Center, Box 596, 75124 Uppsala (Sweden)

[c] Dr. Y. Karimi-Nejad Solvay Pharmaceuticals $\mathrm{GmbH}$ Hans-Böckler-Allee 20, 30173 Hannover (Germany)

[d] Dr. A. Stary, ${ }^{+}$Prof. Dr. G. Vriend Centre for Molecular and Biomolecular Informatics Nijmegen Centre for Molecular Life Sciences Radboud University Nijmegen P.O. Box 9010, 6500 GL Nijmegen (The Netherlands)

$\left.{ }^{+}\right]$Current address: Department for Pharmacology and ToxicologyUniversity of Vienna, Althanstrasse 14, 1090 Vienna (Austria)

Supporting information for this article is available on the WWW under http://dx.doi.org/10.1002/cmdc.200900461. 
errors. Our underlying assumption is that a reliable model (based on a correct alignment) should not fail with any assessment method, should have reasonable stability in MD simulations, and should be suitable to study drug-receptor interactions in a qualitative and possibly quantitative manner. Only one out of the seven tested hERG models fulfilled these criteria. This model was confirmed by a recent mutation scanning experiment ${ }^{[1]}$ that is consistent with the alignment underlying this model.

\section{Results}

\section{Models of the pore-forming domain}

hERG models were built by using $\mathrm{K}_{\mathrm{v}} \mathrm{AP}$ as the template; they include S5 segments, the P-helix re-entrant loops, and S6 segments (Figure 1 B). Alignments 2-6 were extracted from the literature, ${ }^{[11-14,16]}$ and alignments for models 1 and 7 were added for completeness. During the modeling process, fourfold symmetry was applied. The S5 turret helices and voltage-sensing helices $\mathrm{S1-S4}$ were omitted from the models as described previously, ${ }^{[13]}$ facilitating comparison between different hERG models. Except for model 2, which was downloaded from the Schrödinger homepage (http://www.schrodinger.com/productpage/14/6/75/), the backbone of hERG models was not manually adjusted, as has been described for some models. ${ }^{[11,13]}$ Farid et al. ${ }^{[11]}$ describe rotational movements of backbone torsion angles for residues G648 (S6) and G572 (S5), which result in an increased pore size relative to $\mathrm{K}_{\mathrm{v}} A P$.

Figure $1 \mathrm{~B}$ shows the pore-forming domain of a $\mathrm{hERG}$ model with residues critical for drug block highlighted. Consistent with alanine scan experiments (for examples, see references $[10,25])$, the side chains of residues T623, S624, Y652, and F656 of all seven hERG models are oriented toward the pore, enabling direct interactions with blockers.

In hERG, the degree of identity with templates of known structure varies for different segments. The "signature sequence" of the selectivity filter TVGYG is highly similar between potassium channels of known structure (Mlotik, ${ }^{[26]}$ $\mathrm{KcsA}^{[20]} \mathrm{MthK}^{[21]} \mathrm{K}_{\mathrm{v}} 1.2{ }^{[23,24]}$ and $\mathrm{K}_{\mathrm{v}} \mathrm{AP}^{[22]}$ ) and hERG, which contains a slightly modified SVGFG motif. An unambiguous alignment of 56 segments is possible due to the presence of a highly conserved glycine hinge and reasonable sequence identities of $39 \%$ between S6 segments. The outer helices formed
A)

Mlotik
KcsA
MthK
Kv1.2
KvAP
m1 $\quad( \pm 0)$
m2 $\quad(+4)$
m3
m4 (+2)
m5 (+1)
m6 $\quad(-2)$
m7 $\quad(-6)$

Mlotik

KcsA

MthK

Kv1.2

KvAP

$m 1-m 7$

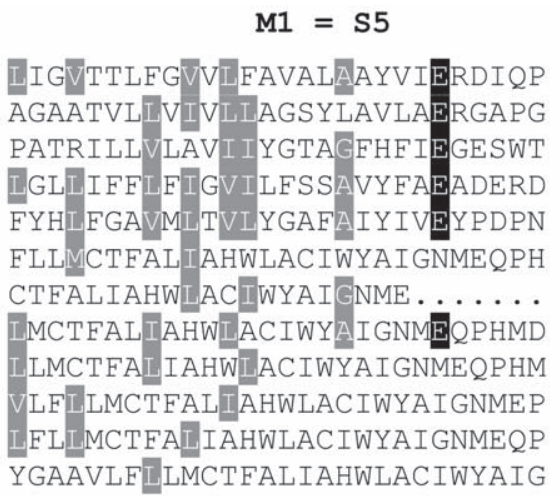

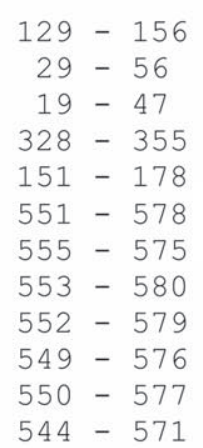

$\mathrm{P} / \mathrm{SF}$

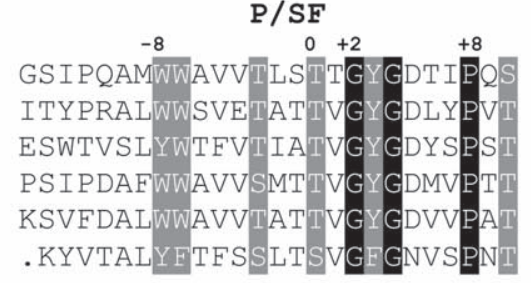

$160-185$

$60-85$

$44-69$

$359-384$

$182-207$

$610-634$

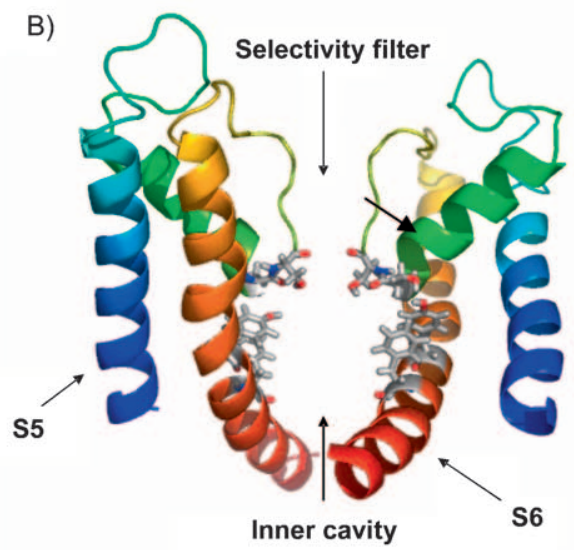

$\begin{array}{llr}\text { Mlotik } & \text { FAGRVLAGAVMMSGIGIFGLWAGILATGFYQE } & 186-217 \\ \text { KcsA } & \text { LWGRLVAVVVMVAGITSFGLVAALATWFVGR } & 86-117 \\ \text { MthK } & \text { PLGMYFIVILIVLGIGTFAVAVERLLEFL... } & 70-98 \\ \text { Kv1.2 } & \text { PIGKIVGSLCAIAGVLTIALPVPVIVSNFNY } & 388-415 \\ \text { KvAP } & \text { IGGKVIGIAVMLTGISALTLLIGTVSNMFQKI } & 208-238 \\ \text { m1 - m7 } & \text { NSEKIFSICVMLIGSLMYASIFGNVSAIIQRL } & 635-666\end{array}$

Figure 1. A) Structural alignment of the pore-forming domains of Mlotik ${ }^{[23]}(P D B$ ID: $3 B E H), K_{c s} A^{[20]}\left(1 K_{4} C\right), M_{t h K}{ }^{[21]}\left(1 L^{2 N Q}\right), K_{v} 1.2^{[23,24]}(2 R 9 R)$, and $K_{v} A P^{[22]}$ (1ORQ) channels with hERG; m1-m7 denote the various hERG alignments with $\mathrm{K}_{v} A P$. Alignments for models 2-6 were extracted from the literature (see Experimental Section). Numbers in parentheses indicate the shift of helix S5 relative to the alignment of $\mathrm{m} 1$ (arbitrarily taken as reference). Identical residues in all sequences are boxed in black, and similar residues are boxed in gray. Numbers above the alignment indicate residues that are part of the pore loop signature sequence present in most potassium channels; ${ }^{[37]}$ E637 is boxed. B) Side view of two diagonal subunits of the pore-forming domain of hERG with key residues T623, S624, Y652, and F656 important for drug binding shown in stick representation. Helices S5 are colored blue, P-helices are colored green, the selectivity filter loops are yellow, and the $\mathrm{S} 6$ segments are colored red. 
by S5 segments are more difficult to align due to the low sequence identity between $\mathrm{hERG}$ and the $\mathrm{K}_{\mathrm{v}} \mathrm{AP}$ template and generally between different potassium channels (Figure 1A). The pore domains of the potassium channel crystal structures display poor conservation at the sequence level (21-38\%), but the correct alignment can be obtained by generating structural alignments. They reveal a remarkable similarity in 3D space, justifying the use of currently available crystal structures such as $\mathrm{K}_{\mathrm{v}} \mathrm{AP}, \mathrm{MthK}, \mathrm{K}_{\mathrm{v}} 1.2$, or $\mathrm{KcsA}$ to generate hERG models. All five structures have a conserved glutamate residue at the extracellular end of S5 that interacts with backbone amide nitrogen atoms and the hydroxy group of a conserved threonine in the loop connecting the selectivity filter with $\mathrm{S} 6$ segments. Mutation of the corresponding residue in Shaker (E418) revealed an important role of this charged residue for normal gating. ${ }^{[27]}$ Published alignments for hERG show considerable variation in S5 with relative shifts of this helix of more than one helical turn in the $\mathrm{N}$ - or C-terminal direction (see Figure $1 \mathrm{~A}$ ).

The alignment of model 3 suggests that E575 of hERG might stabilize the post-selectivity filter in a similar way as observed in the crystal structures. However, mutations of E575 to cysteine or positively charged lysine are well tolerated ${ }^{[28]}$ indicating a different role for E575 in hERG. All hERG models have a conserved glutamate (E637) at the top of the S6 helix, which might provide hydrogen bonds that stabilize this part of the channel (see also Stansfeld et al. ${ }^{[13]}$ ). This hypothesis is consistent with experimental observations that mutations of E637 to lysine lead to poor channel expression. Furthermore, mutations at this position are linked to LQTS, ${ }^{[29]}$ underscoring the functional importance of residue E637.

\section{Intra- and inter-subunit interactions in various hERG models}

In all seven hERG models, hydrogen bonds between Y652 and S649 (both S6) from neighboring subunits are observed. Except for models 2 and 7, these interactions are maintained during MD simulations. Hydrogen bonds are also formed between E637, located at the extracellular end of segment S6, and the backbone of $\mathrm{N} 633$ and backbone and side chain of T634, located at the post-selectivity filter loop. These interactions remain stable in simulations in models $4-7$, but are lost in models 1-3. Model 6 contains additional inter-domain hydrogen bonds between Y667 (S6) and T556 (S5), which are preserved in MD simulations.

\section{Aromatic-aromatic interactions}

In the hERG pore domain, 15-19 aromatic residues per subunit are present, depending on the alignment used. Five to eight of these are present in S5 segments. Alignment differences lead to different interaction patterns between aromatic side chains in the models (Table 1 and Figure 2). Model 1 has the greatest number of favorable aromatic-aromatic interactions, with four pairs between residues of S5, the P-segment, and S6 from the same subunit. Additionally, a cluster of three aromatic side chains between S5, the P-helix, and the selectivity filter from the neighboring subunit is present. In model 6, two clusters

\begin{tabular}{|c|c|c|c|c|c|}
\hline Model & F551 & F557 & H562 & W563 & W568 \\
\hline $\mathrm{m} 1$ & - & Y667 (S6) & F619 (P) & Y611 (P) & $\begin{array}{c}\text { F617 (P) } \\
\text { F627 (SF) } \\
\text { inter }^{[a]}\end{array}$ \\
\hline $\mathrm{m} 2$ & - & - & - & F619(P) & F640 (S6) \\
\hline $\mathrm{m} 3$ & $\mathrm{Nn}^{[b]}$ & - & - & Y611 (P) & - \\
\hline $\mathrm{m} 4$ & $N n^{[b]}$ & - & - & - & - \\
\hline m5 & - & F619 (P) & - & F617 (P) & - \\
\hline $\mathrm{m} 6$ & - & $\begin{array}{l}\text { Y652 (S6) } \\
\text { F619(P) }\end{array}$ & Y611 (P) & - & - \\
\hline $\mathrm{m} 7$ & Y667(S6) & - & F640 (S6) & $\mathrm{F} 617(\mathrm{P})$ & - \\
\hline
\end{tabular}

with three aromatic side chains and one paired interaction between S5 and the P-segment exist. In model 7, three pairs of aromatic-aromatic interactions are identified, while models 2 and 5 possess only two aromatic pairs, between S5 residues and neighboring segments. Models 3 and 4 contain only one aromatic pair between $\mathrm{S} 5$ residues and other segments.

\section{Model evaluation-quality assessment programs}

To identify the correct alignment, various quality assessment methods have been applied. Because most of the methods have been developed for globular proteins and use statistical potentials from known structures, we validated the suitability of the selected quality assessment methods for membranespanning potassium channels. The crystal structure of $\mathrm{K}_{\mathrm{v}} 1.2$ was used because it has higher resolution $(2.4 \AA)$ than that of $\mathrm{K}_{v} A P(3.2 \AA)$. $\mathrm{K}_{v} 1.2$ scores well with all methods tested; no low scores are reported. Scores for $\mathrm{K}_{\mathrm{v}} \mathrm{AP}$ are also acceptable, with the exception of Verify3D and Procheck, with which low values are observed. To assess the discriminative power of these programs to distinguish correctly aligned from misaligned models, a synthetic test set consisting of $\mathrm{K}_{\mathrm{v}} 1.2$ models with shifted S5 segments (one helical turn in steps of one amino acid in both directions, $\mathrm{m}-1$ denotes a shift toward the $\mathrm{C}$ terminus) was built, and the quality screened. Results are summarized in Table 2 A. Only four out of eight methods (WHAT_CHECK Packing 2, Prosall, ProQres LG, and DFIRE) ranked the crystal structure highest. Most shifts in S5 do not noticeably influence the results obtained with ProQres, Procheck, or ModFOLD. The strongest variations were obtained with Verify3D, with which the best model (alignment shifted two residues toward the $\mathrm{N}$ terminus) scored $\sim 23 \%$ higher than the worst model (alignment shifted three residues toward the $C$ terminus), but the crystal structure was ranked only third best (Table $2 \mathrm{~A}$ ).

Table 2B summarizes the results obtained with Verify3D, Procheck, WHAT_CHECK, Prosall, ProQres, DFIRE, and ModFOLD 

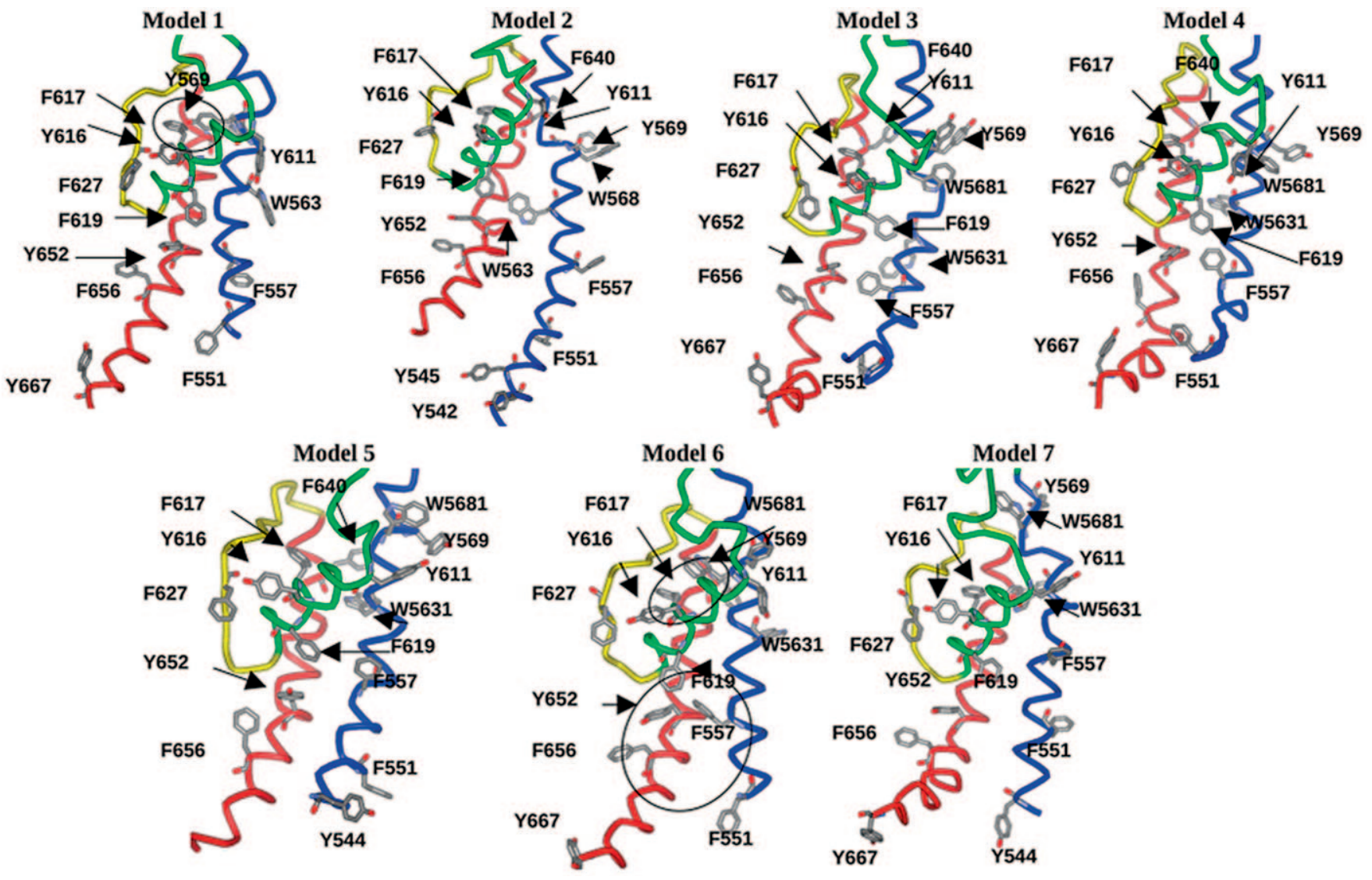

Figure 2. Details of aromatic-aromatic interactions in the various hERG models 1-7: Shown in each case is a side view of averaged hERG coordinates after 10 ns MD simulations, with aromatic side chains (Phe, Tyr, Trp) shown in stick representation. S5 segments are colored blue, P-helices are shown in green, the selectivity filter is yellow, and S6 segments are colored red. For model 1, favorable aromatic-aromatic interactions between segment S5, the P-helix, and S6, stabilizing the selectivity filter are circled. For model 6, only favorable aromatic-aromatic interactions between S5 and other segments are observed. Stabilizing interactions in the selectivity filter region and the pore region are circled.

\begin{tabular}{|c|c|c|c|c|c|c|c|c|c|c|c|c|c|c|c|c|}
\hline \multirow{3}{*}{$\begin{array}{l}\text { A) } \\
\text { Model } \\
\text { KvAP }\end{array}$} & \multirow{2}{*}{\multicolumn{2}{|c|}{ Prosa2003 }} & \multirow{2}{*}{\multicolumn{2}{|c|}{ Verify3D }} & \multirow{2}{*}{\multicolumn{2}{|c|}{$\begin{array}{l}\text { ProQres } \\
\text { LG score }\end{array}$}} & \multirow{2}{*}{\multicolumn{2}{|c|}{$\begin{array}{l}\text { ProQres } \\
\text { MaxSub }\end{array}$}} & \multirow{2}{*}{\multicolumn{2}{|c|}{$\begin{array}{c}\text { WHAT_CHECK } \\
\text { Packing } 2\end{array}$}} & \multirow{2}{*}{\multicolumn{2}{|c|}{ DFIRE/res. }} & \multirow{2}{*}{\multicolumn{2}{|c|}{$\begin{array}{c}\text { Procheck } \\
\phi / \psi\end{array}$}} & \multirow{2}{*}{\multicolumn{2}{|c|}{ ModFOLD }} \\
\hline & & & & & & & & & & & & & & & & \\
\hline & -5.57 & - & 68.88 & - & 5.431 & - & 0.201 & - & -0.447 & - & -100.55 & - & 83.5 & - & 0.80 & - \\
\hline Kv1.2 & -5.55 & 6 & 76.08 & 3 & 6.880 & 1 & 0.636 & 2 & -0.172 & 1 & -109.59 & 1 & 92.8 & 9 & 0.89 & 6 \\
\hline $\mathrm{tm}+1$ & -3.67 & 5 & 71.76 & 5 & 5.977 & 5 & 0.617 & 3 & -0.736 & 8 & -103.61 & 4 & 97.6 & 1 & 0.93 & 2 \\
\hline $\mathrm{tm}+3$ & -3.41 & 8 & 66.41 & 6 & 6.648 & 2 & 0.573 & 7 & -0.529 & 3 & -103.20 & 5 & 97 & 2 & 0.91 & 5 \\
\hline $\mathrm{tm}+4$ & -3.07 & 9 & 55.98 & 9 & 5.021 & 8 & 0.472 & 9 & -0.699 & 6 & -102.27 & 8 & 95.5 & 6 & 0.86 & 7 \\
\hline $\mathrm{tm}-1$ & -4.1 & 1 & 75.83 & 4 & 6.207 & 4 & 0.600 & 5 & -0.280 & 2 & -105.69 & 2 & 94.9 & 7 & 0.93 & 1 \\
\hline $\mathrm{tm}-2$ & -3.7 & 4 & 77.35 & 2 & 6.403 & 3 & 0.679 & 1 & -0.652 & 4 & -104.94 & 3 & 96.1 & 4 & 0.92 & 4 \\
\hline $\mathrm{tm}-3$ & -3.71 & 3 & 62.85 & 8 & 5.655 & 7 & 0.583 & 6 & -0.696 & 5 & -102.89 & 6 & 94.9 & 8 & 0.82 & 8 \\
\hline $\mathrm{tm}-4$ & -3.42 & 7 & 66.41 & 6 & 4.723 & 9 & 0.494 & 8 & -0.858 & 9 & -100.79 & 9 & 97 & 3 & 0.82 & 9 \\
\hline \multicolumn{17}{|l|}{ B) } \\
\hline $\mathrm{m} 1$ & -4.01 & 2 & 51.46 & 5 & 4.985 & 1 & 0.145 & 6 & -1.613 & 4 & -103.46 & 3 & 93.2 & 2 & 0.65 & 3 \\
\hline$m 2^{[10]}$ & -2.09 & 5 & 39.22 & 7 & 1.622 & 7 & -0.081 & 7 & -0.606 & 1 & -88.69 & 7 & 89.2 & 7 & 0.32 & 7 \\
\hline$m 3^{[11]}$ & -2.41 & 4 & 64.38 & 3 & 4.266 & 3 & 0.380 & 2 & -2.078 & 7 & -99.67 & 6 & 92.8 & 3 & 0.38 & 6 \\
\hline $\mathrm{m} 4^{[15]}$ & -2.01 & 6 & 69.77 & 2 & 3.922 & 4 & 0.365 & 3 & -2.057 & 6 & -99.69 & 5 & 93.4 & 1 & 0.38 & 5 \\
\hline$m 5^{[12]}$ & -1.99 & 7 & 50.96 & 6 & 3.520 & 5 & 0.276 & 5 & -2.016 & 5 & -100.16 & 4 & 92.2 & 5 & 0.41 & 4 \\
\hline$m 6^{[13]}$ & -3.58 & 3 & 79.2 & 1 & 4.377 & 2 & 0.408 & 1 & -0.855 & 2 & -105.92 & 2 & 91.2 & 6 & 0.68 & 1 \\
\hline $\mathrm{m} 7$ & -4.75 & 1 & 57.84 & 4 & 3.418 & 6 & 0.339 & 4 & -1.530 & 3 & -115.57 & 1 & 92.4 & 4 & 0.68 & 1 \\
\hline
\end{tabular}


A)

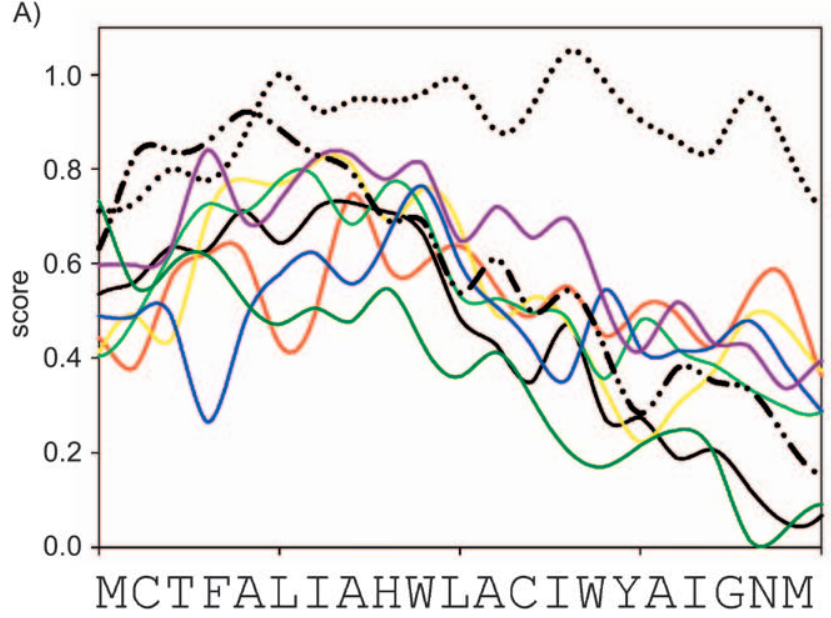

B)

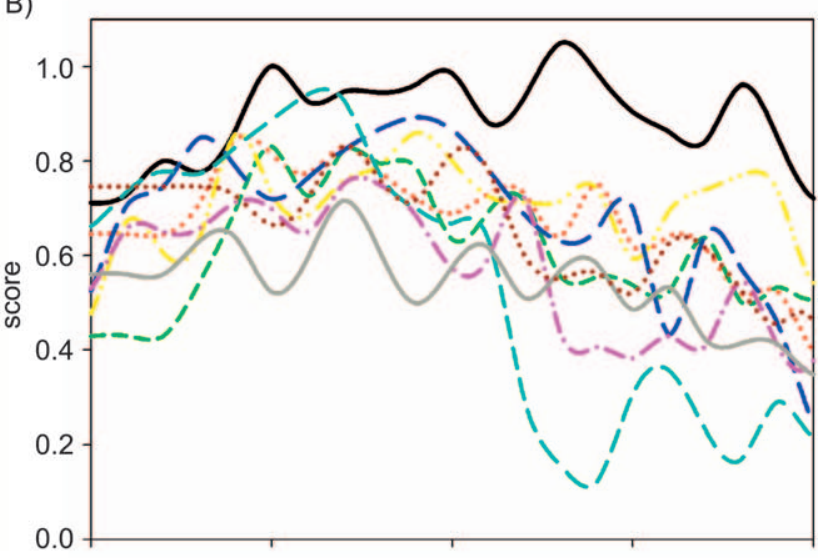

LLIFFLFIGVILFSSAVYFAE

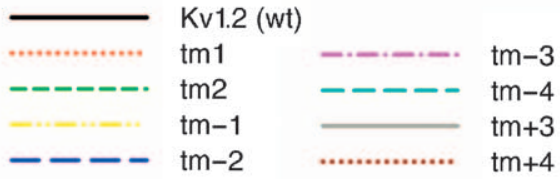

Figure 3. Local model quality assessment: A) Local quality of segment $\mathrm{S} 5$ of all seven hERG models, as well as crystal structures $\mathrm{K}_{\mathrm{v}} \mathrm{AP}$ and $\mathrm{K}_{\mathrm{v}} 1.2$, as assessed with the method developed by Fasnacht et al. ${ }^{[30]}$ The amino acid sequence of helix S5 is shown below the plot. Higher scores indicate higher quality. B) Local quality of $\mathrm{K}_{\mathrm{v}} 1.2$ and synthetic test models.

for the hERG models. Models are ranked from 1 to 7 , with 1 denoting the best-ranked model in each category. Low values are shown in bold. Static checks show reasonable scores for model 1, with the exception of Verify3D and ProQres MaxSub score, which are lower than expected for good structures. Scores for model 2 are very low, indicating severe problems. Models 3-5 have similar quality, with problematic packing values and very low scores with ModFOLD. Scores for model 6 are favorable, and none of the methods suggest structural problems. Scores for model 7 are acceptable except for Verify3D, which reports a low value (see Table $2 \mathrm{~B}$ ). The ranking of hERG models is less clear, and lower scores are obtained for all models relative to the crystal structures. Model 6 scores slightly better than models 3 and 4, model 1 has intermediate quality, and models 5 and especially 2 score lowest.

The local quality of S5 segments was evaluated with a method developed by Fasnacht et al. ${ }^{[30]}$ The results are summarized in Figure 3. $\mathrm{K}_{\mathrm{v}} 1.2$ shows the highest quality throughout the whole segment. This method shows a clear distinction between different alignments in our synthetic test set, with the largest differences observed for the second half of S5. The results for the hERG models are less straightforward to interpret, yet a similar quality trend emerges. Models 1 and 6 are again among the best, models 3,4 , and 5 show intermediate qualities, and models 2 and 7 score lowest.

\section{Molecular dynamics simulations}

hERG models were examined using MD simulations with the protein models embedded in a POPC lipid bilayer, and each simulation was repeated twice with different initial velocities. The root mean square deviation (RMSD) of a protein from its starting coordinates as a function of time is routinely used as a measure of its structural stability. Figure $4 \mathrm{~A}$ shows the RMSD values of the backbone atoms of the hERG models. The stability was compared with $\mathrm{K}_{\mathrm{v}} \mathrm{AP}$ and $\mathrm{K}_{\mathrm{v}} 1.2$ (Figure $4 \mathrm{~B}$ ), which display RMSD values in the range of $0.16-0.2 \mathrm{~nm}$. hERG models 1 , 3,5 , and 6 have only slightly higher RMSD values in the range of $0.2-0.25 \mathrm{~nm}$. Models 2, 4, and 7 are less stable, with RMSD values in the range of $\sim 0.35 \mathrm{~nm}$ after $10 \mathrm{~ns}$. Furthermore, the RMSD curves of these models are still rising, indicating that these systems have not yet found local minima. The results for the extended simulations of model $1(60 \mathrm{~ns})$ and model 6 (100 ns) are shown in Figure 4C.

Table 1 lists aromatic-aromatic interactions in the various hERG models prior to MD simulations. Important changes are observed during MD simulations; these variations are of particular interest, as they may directly influence drug blocking. Figure 5 summarizes the distance measurements between F656 from various subunits as a function of time. The distance is defined as the distance between the geometric centers of the phenyl rings.

Models 1 and 5 show very short distances between adjacent phenyl rings. Models 3, 4, and 7 show values in the range of 1-1.5 nm, and model 2, which has been adjusted manually to increase the pore size (for details, see reference [11]) and model 6 (no modification) display larger inner cavities, with distances between phenyl rings in the range of $2-2.5 \mathrm{~nm}$. In models 3 and 5, the inner cavity "collapses" during MD simulations; F656 residues from all four subunits cluster tightly. 
A)

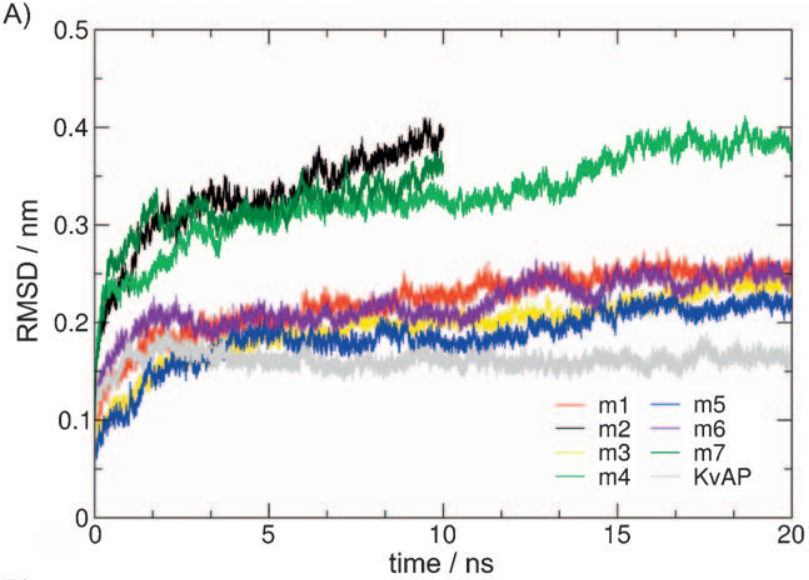

B)

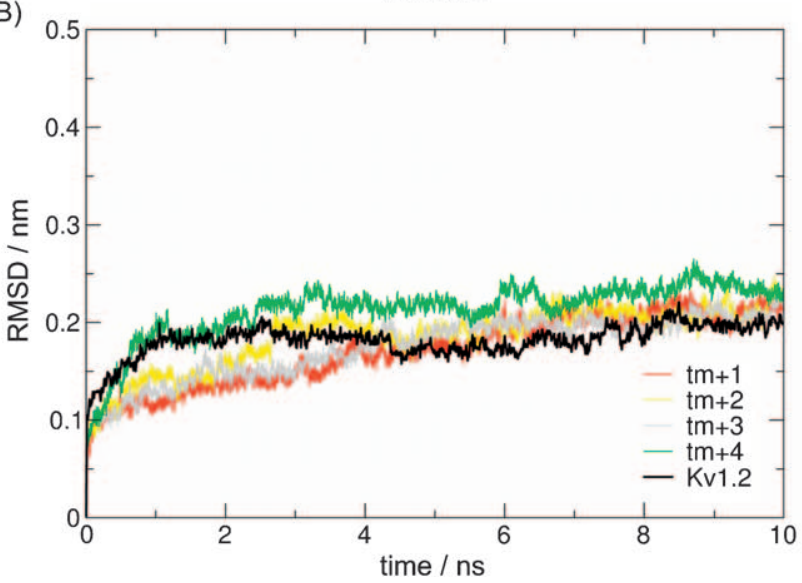

C)

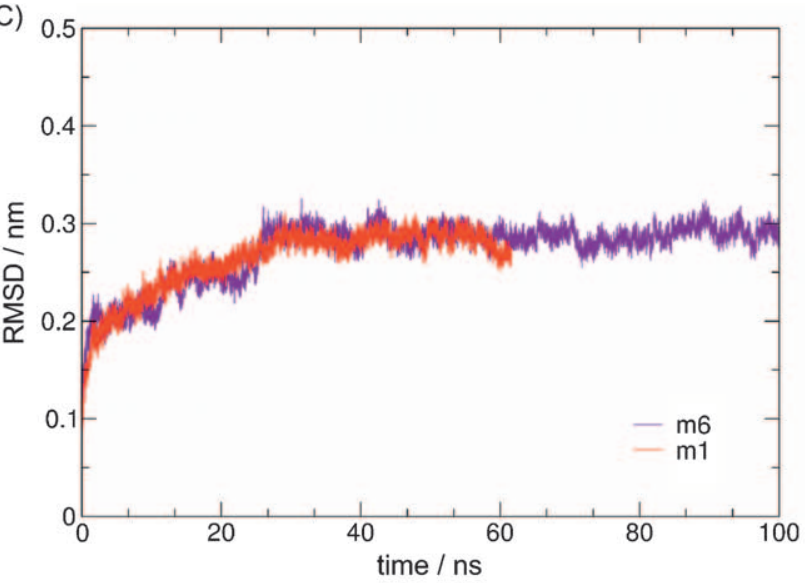

D)

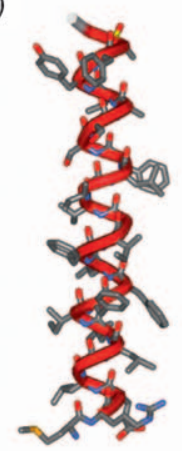

Figure 4. RMSD values of the various $h E R G$ models, $K_{v} A P$, and $K_{v} 1.2$ plus synthetic test set: $A$ ) Results from MD simulations with $h E R G$ models embedded in a POPC lipid bilayer under physiological ion concentrations are shown. All simulations were repeated twice, as described in the Experimental Section. The RMSD plots for all backbone atoms of $h E R G$ models $m 1-m 7$ are shown. B) The stability of $\mathrm{K}_{\mathrm{v}} 1.2$ and different $\mathrm{K}_{\mathrm{v}} 1.2$ test models during MD simulations. C) Simulations for models 1 and 6, which performed best in our static assessments, were simulated for 60 and $100 \mathrm{~ns}$, respectively. D) Ribbon and sticks representation of helix $\mathrm{S} 5$ from the $\mathrm{K}_{\mathrm{v}} 1.2$ crystal structure.

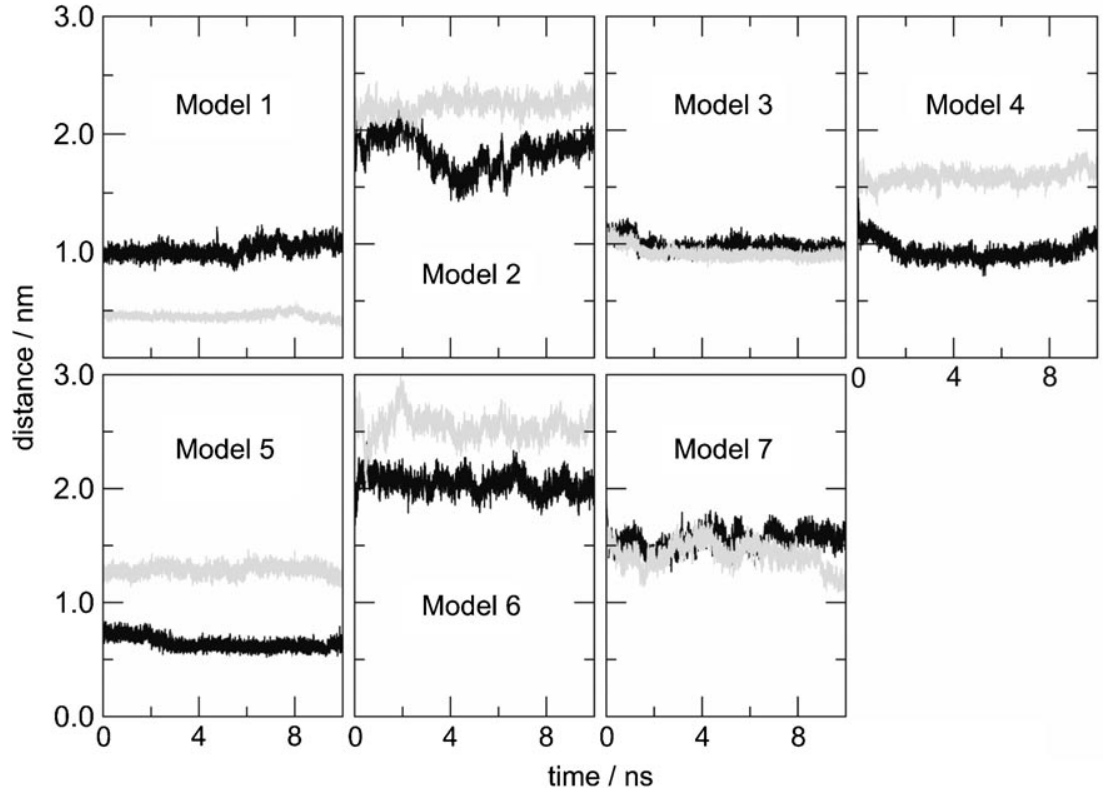

Figure 5. Influence of the conformation of F656 on hERG pore size: The distances between the geometric centers of F656 phenyl rings of adjacent subunits are shown as a function of time. Models 1, 3, 4, and 5 show very narrow pores, with F656 residues from several subunits interacting directly with each other, thereby "collapsing" the pore. Larger distances between adjacent F656 rings are observed for models 2 and 6, while model 7 displays an intermediate pore size.

\section{Experimental validation of hERG models}

Recently, Ju et al. ${ }^{[1]}$ studied the structure and function of helix S5 in detail using a combination of NMR and mutagenesis studies. These data were not included in the model building and evaluation process and can therefore be used to cross-validate the results of our study. Figure 6 shows a side view of one domain of each hERG model, with residues perturbing inactivation $^{[1]}$ shown as spheres. According to Ju et al., ${ }^{[1]}$ these residues should point toward the inner S6 helix. Using the alignment of model 1, only F551 and the side chain of W568 are orientated toward the pore. In model 2, the situation is even 

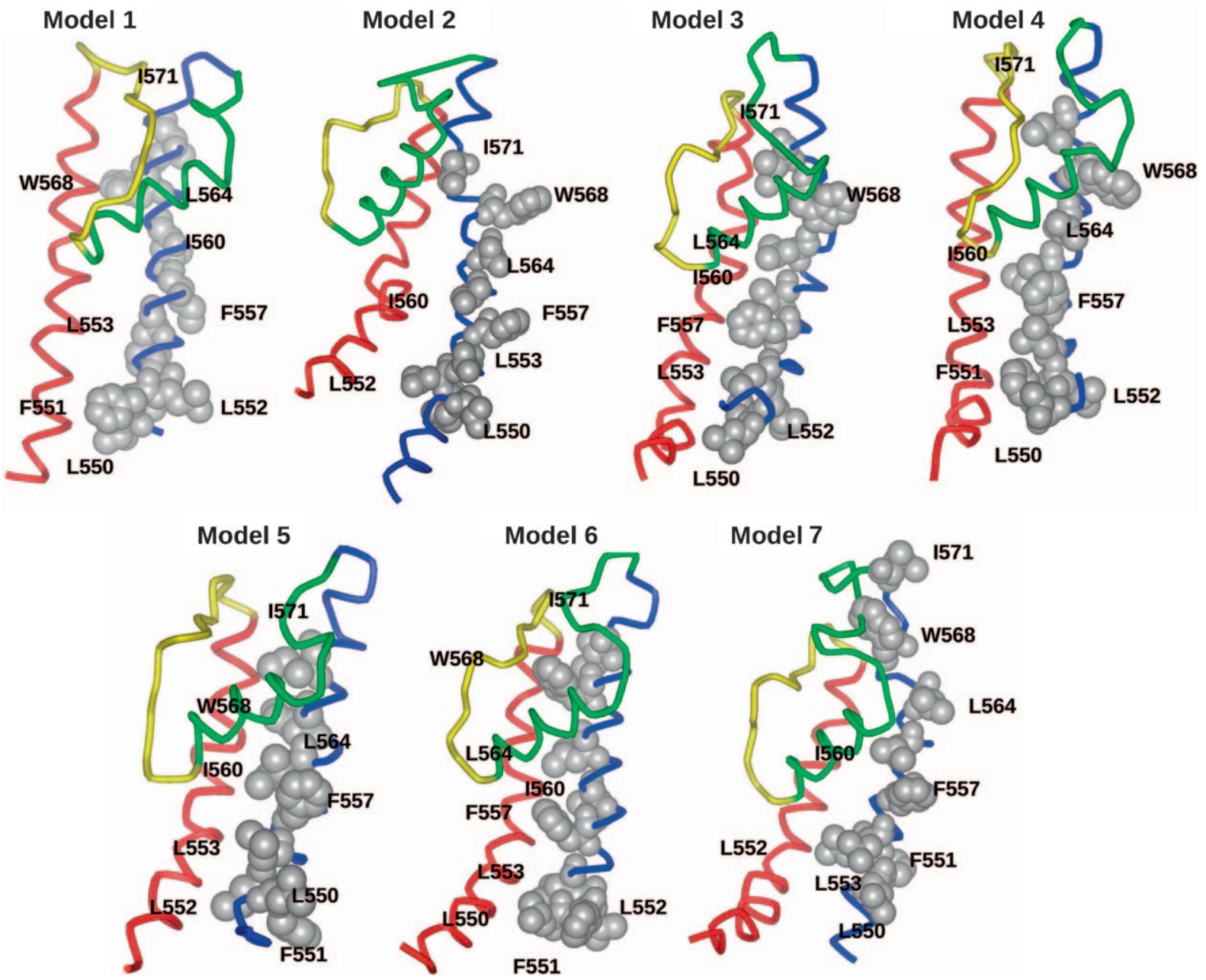

Figure 6. Agreement of the various hERG models with the results of a recent mutagenesis study: Shown are ribbon representations of one subunit of each hERG model, with residues perturbing the inactivation shown as spheres; these residues are thought to be predominantly pore-facing. ${ }^{[1]}$

worse; except for L552 all inactivation-perturbing residues are orientated toward the voltage-sensing domain. In model 3 , only residues L551 and W568 face the opposite side of the pore. In model 4, two of the inactivation-perturbing residues, L552 and W568, are orientated toward the voltage-sensing domain. In model 5, residues L550 and F551 cannot interact with the inner pore helix. Model 6 fits the experimental data of Ju et al. ${ }^{[1]}$ best, as only the side chain of L552 does not face toward helix S6. The alignment of model 7 is shifted extensively, and residues W568 and 1571 are not in the transmembrane core, but in the extracellular loop region. Additionally, the side chains of F551 and L564 are orientated toward the voltagesensing domain.

F557 on S5 was found by Ju et al..$^{[1]}$ to exert an especially pronounced facilitating effect on the inactivation of hERG. Mutation of this residue to alanine decreases the energy barrier to inactivation by $\sim 1.5 \mathrm{kcal} \mathrm{mol}^{-1}$. In our model 6 , the side chain of F557 is situated next to that of Y652 of S6, and both aryl rings undergo a direct $\pi-\pi$ stacking interaction in the model which remains stable in MD simulations. It is conceivable that F557 influences the rotameric state of the Y652 side chain in hERG. In turn, Y652 stacks in a parallel fashion onto F656 and thus stabilizes its conformation. The aromatic residues on S6 have been shown to be strongly involved in inactivation and/ or drug binding. ${ }^{[31,32]}$ In further support of model 6, residues within the helical part of S5 (F551, L559, and W563) experimentally found to facilitate activation gating are orientated toward the voltage-sensing domains. A strong interaction with the voltage-sensing domain thus appears highly plausible, as was suggested by Ju et al. ${ }^{[1]}$

\section{Interaction of different hERG models with high-affinity blockers}

A large amount of experimental data on various drugs is available for hERG. Using these data to distinguish between various S5 alignments is not straightforward, as mutational studies focus on the inner S6 segments and the bottom of the selectivity filter, where drug binding occurs. However, the alignment of both SF and S6 segments are identical for all seven hERG models. An indirect evaluation based on proposed drug binding modes might be possible. For this purpose, we docked the high-affinity blocker (+)-cisapride into the cavities of all seven hERG models. Cisapride was selected because the importance of the positioning of the aromatic residues in S6 has been demonstrated experimentally (for examples, see referen- 
ces [31,33]). Additionally, (S)-terfenadine and MK-499 were docked into the two best-ranked models, model 1 and model 6. FlexX and GOLD, with standard parameters, were used to analyze hERG-drug interactions. Both programs require the definition of residues that line the binding cavity. Therefore, residues Y652, F656, T623, and S624 from all four subunits were chosen as starting points for docking. Observations in the docking of cisapride are described in detail for all seven models (Figure 7). Additionally, details of the docking results for (S)-terfenadine and MK-499 are presented for model 6, but not for model 1, because docking was not successful in the latter case. Minimized averaged structures after $10 \mathrm{~ns}$ MD simulations as well as structures prior to MD simulations served as starting conformations for docking, and only the 20 top-scoring poses of each model were analyzed. For models 1 , 3 , and 5, no reasonable docking poses within the cavity could be obtained using minimized average structures after MD simulations. Therefore, additional minimized snapshots of the tra- jectory were extracted for docking. However, most snapshots were unsuitable for docking, with the exception of the starting coordinates, which provided large enough cavities for drug docking (data not shown).

The docking poses for the starting structure of model 2 are similar as described in Farid et al. ${ }^{[11]}$ In agreement with this study, cation $-\pi$ interactions were not predicted. Docking poses obtained from the average structure after $10 \mathrm{~ns}$ MD simulation changed due to considerable deviations from the starting structure (Figure 4), and a hydrogen bond between the methoxy group of the benzamidine ring and the hydroxy group of S649 (S6) was predicted. Aromatic interactions of Y652 and F656 to three subunits are observed; however, instead of interactions to three $\mathrm{F} 656$ and two $\mathrm{Y} 652$ residues from different subunits, interactions to two $\mathrm{Y} 652$ and three $\mathrm{F} 656$ residues are predicted for the final MD structure.

Docking scores obtained for model 4 are considerably lower than the results for model 2. Again, due to large deviations
A)

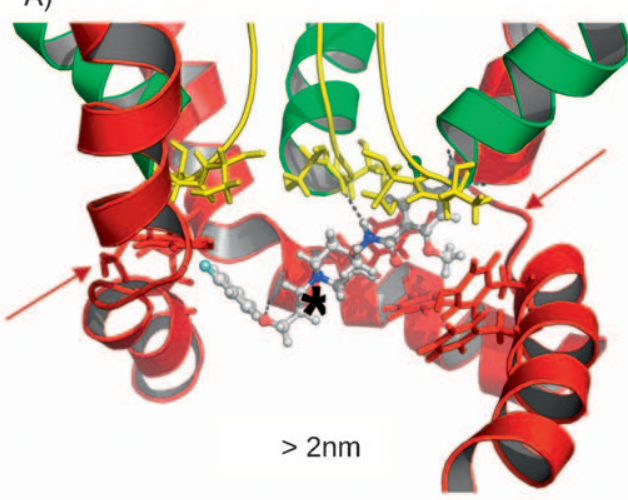

D)

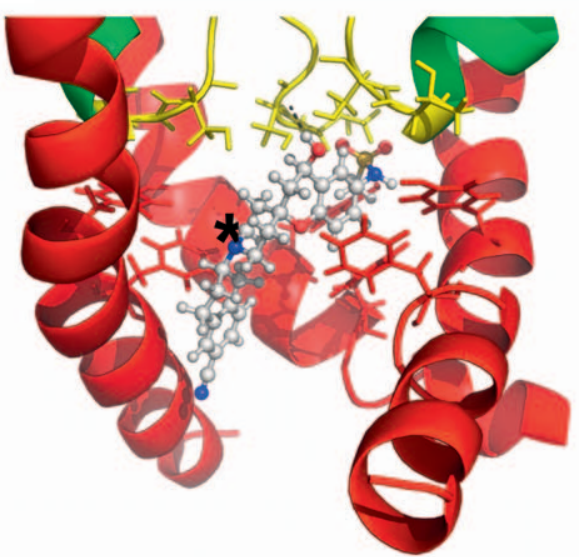

B)
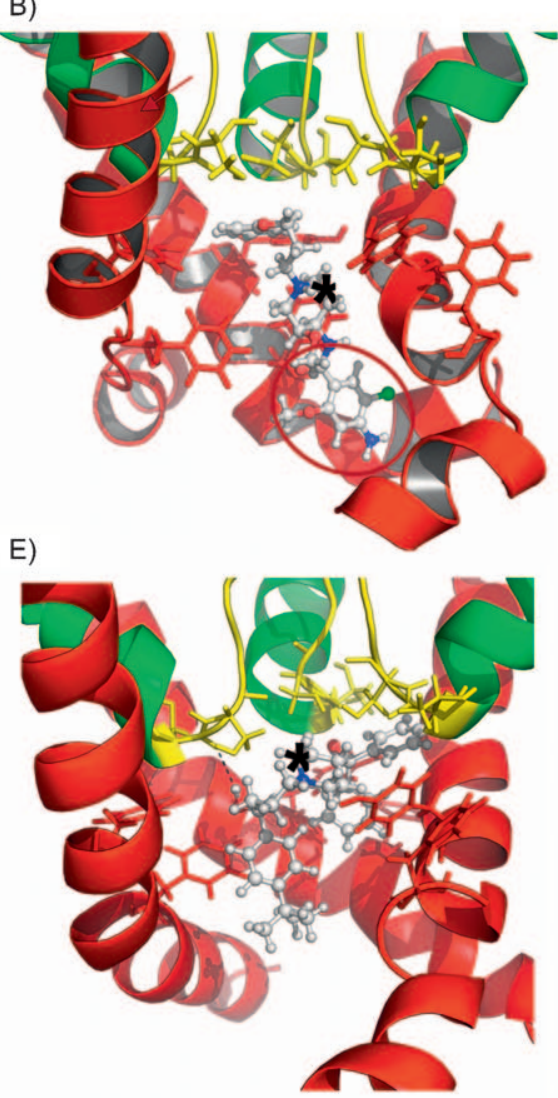

C)

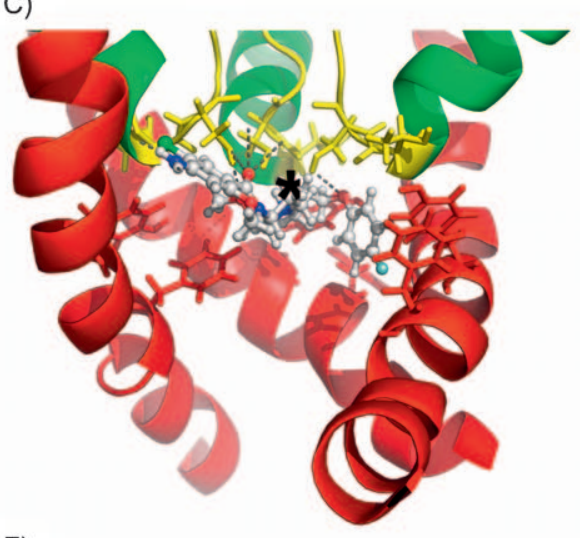

F)

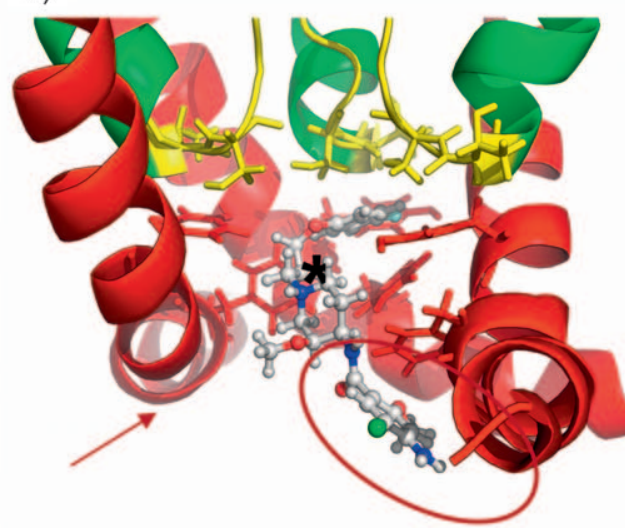

Figure 7. Interactions of the various hERG models with the high-affinity blocker cisapride. Snapshots of the best-ranked docking poses for models 2, 4, 6, and 7 are shown. Helices S6 (red), P-helix (green), and the selectivity filter (yellow) of two diagonal subunits are shown in ribbon representation, and residues T623, S624, Y652, and F656 are shown as sticks and are colored according to the segments to which they belong. The location of the positively charged nitrogen atom is marked (*). A) Red arrows indicate the distorted geometry of the $\alpha$ helix in this region, which is most likely a consequence of the interventions at G648. Hydrogen bonds are shown as black dotted lines. B) Orientation of cisapride in the averaged coordinates (10 ns) of model 4 . Helices S6 are kinked, influencing the shape of the binding cavity. The benzamidine ring of cisapride (circled in red) does not interact with any experimentally determined residues, but is oriented toward the intracellular cavity. C) Cisapride interactions with averaged coordinates of model 6 after 10 ns MD simulation. Hydrogen bonds are shown as black dotted lines. D) Interactions of MK-499 with the averaged coordinates of model 6 after 10 ns. The hydrogen bond to S624 is shown as a black dotted line. E) Terfenadine hERG interactions for model 6, after $10 \mathrm{~ns}$ MD simulation, are shown. Hydrogen bonds to the selectivity filter residues are shown as black dotted lines. F) Best docking pose obtained for the averaged coordinates of model 7 after 10 ns MD simulation. Helices S6 are considerably bent, influencing the shape of the cavity. Similar to model 4, the drug is orientated perpendicular with respect to the benzamidine ring of cisapride oriented toward the intracellular side (circled). 
from the starting structure, (+)-cisapride-hERG interactions changed considerably from the starting structure to the averaged structure after $10 \mathrm{~ns}$ MD simulation. Only results for the averaged structure are described in detail in the following section, as the cavities in all starting structures, except for model 2, are very similar. No hydrogen bonds between (+)-cisapride and any polar residues in the cavity are predicted. Interactions to five aromatic side chains are predicted: three to Y652 and two to F656. The benzamidine ring interacts via parallel displaced $\pi-\pi$ stacking from neighboring Y652 residues, while hydrophobic contacts are predicted to F656 residues.

Docking into starting and end coordinates of model 6 yielded reasonable scores. Simultaneous interactions between six aromatic side chains and (+)-cisapride are observed. The fluorophenoxy ring is flanked by two Y652 rings and one F656 ring which interact via $T$-shaped $\pi-\pi$ stacking. Furthermore, a hydrogen bond is predicted to the oxygen atom of one of the serines (S624) from the base of the selectivity filter. Hydrogen bonds are also predicted between methoxy groups and two additional $\mathbf{S 6 2 4}$ residues and to the backbone carbonyl oxygen atom of one T623 residue. The benzamidine ring interacts with Y652 via parallel displaced $\pi-\pi$ stacking and F656 from the same subunit via T-stacking. There are more favorable interactions between $(+)$-cisapride and model 6 than to any other model.

In contrast to cisapride, only two hydrogen bonds between one hydroxy group of (S)-terfenadine and the side chains of S624 and (a weaker hydrogen bond) to T623 from the same subunit are predicted. This result is consistent with a recent alanine scan reported by Kamiya et al. ${ }^{[25]}$ Additionally, $\pi-\pi$ interactions to three $\mathrm{Y} 652$ residues and one $\mathrm{F} 656$ are predicted. This finding is not completely consistent with the study reported by Imai et al.. ${ }^{[33]}$ in which interactions to diagonal but not adjacent Y652 residues are suggested.

Interactions between model 6 and MK-499 are illustrated in Figure 7. In agreement with a study by Mitcheson et al. ${ }^{[10]} \mathrm{a}$ hydrogen bond between $\mathrm{S} 624$ and the hydroxy group of MK499 is observed. However, the distance is much smaller (1.8 $\AA)$ as proposed by a recent docking study. ${ }^{[34]}$ Similar to the findings of Farid et al., ${ }^{[11]}$ interactions between MK-499 and four aromatic side chains are predicted. No direct contacts to G648 are observed (see Figure 7).

The averaged structure after $10 \mathrm{~ns}$ MD simulation provided a suitable starting conformation to probe $(+)$-cisapride interactions with model 7. Similar to model 4, docking scores are significantly lower than for models 2 and 6 . The binding site in model 7 differs from other models, because bending of the 56 segments in the region between Y 652 and F656 occurs, and the cavity is generally smaller than in models 2 and 6 (see Figures 2 and 5). Again, results for the averaged model after $10 \mathrm{~ns}$ are quite different from the starting coordinates, as the model is not stable in MD simulations. No hydrogen bonds between selectivity filter residues (T623, S624) and (+)-cisapride were observed. Interactions with six aromatic side chains from three different subunits are observed. Aromatic interactions with the fluorophenoxy ring are predicted via parallel stacking between two Y652 residues from neighboring subunits and sandwich stacking occurs to an F656 residue. Surprisingly, no aromatic contacts to the benzamidine ring are present, and the basic nitrogen atom faces toward the intracellular cavity. Taken together, models with collapsed or very narrow inner cavities (models 1, 3, and 5) are not suitable for drug docking. The most favorable docking poses were obtained for model 6, followed by model 2 . Models 4 and 7 have fewer favorable contacts than the other models.

\section{Discussion}

In the present study we investigated the quality and stability of seven different hERG models obtained by homology modeling. Due to the low sequence identity in S5 helices, no consensus about the alignment of this segment has been achieved (Figure $1 \mathrm{~A}$ ). It was therefore a main goal of this study to identify the correct alignment of helix 5 .

The importance of aromatic-aromatic interactions in ion channels has been noted previously. ${ }^{[20,35]}$ In KcsA, residues W67 and W66 form an aromatic cuff, stabilizing the pore loop. ${ }^{[20]}$ This motif ${ }^{[36]}$ which is part of the pore loop signature sequence, is conserved in hERG. The exception is position +8 , which is replaced by a tyrosine residue (see Figure $1 \mathrm{~A}$ ).

A common observation in membrane proteins is the preferential location of aromatic residues, especially Trp and Tyr at the interface between membrane and solvent. ${ }^{[37-41]}$ In the hERG models, aromatic residues are not only at the membrane interface in most models (see Supporting Information figure 1), but there are additional aromatic residues distributed over the protein, making this feature difficult to interpret. Evenly distributed aromatic residues have been observed in the closed conformation of Kirbac1.1. However, a shift toward extra- and intracellular regions has been suggested for the open conformation. ${ }^{[40]}$

There are significant differences between aromatic-aromatic interactions in different hERG models (Table 1 and Figure 2). Interactions between aromatic residues are energetically favorable. ${ }^{[41]}$ One could therefore expect a contribution to the stability of the protein. However, we found limited correlation between the aromatic clusters and the structural stability in MD simulations. Models 1 and 6 are indeed among the most stable, but model 7 is among the least stable (Figure 4). Upon close inspection, an aromatic mismatch between model 7 and the lipid bilayer becomes apparent. With lipid bilayers such as POPC, it is not possible to satisfy the location of most Tyr and Trp residues in the membrane head group region. Several Tyr groups extend into the bulk water. This might well explain the high RMSD values, despite favorable aromatic-aromatic interactions in the protein structure per se.

The assessment methods used show a clear trend among the studied models. Model 2 scores very low with all but one of the tested methods, whereas model 6 is the only model that does not show low values for any of the applied checks. Models 3-5 show intermediate quality, and scores for model 7 are better than average with the exception of Verify3D, which indicates low quality (Table $2 \mathrm{~A}$ ). To evaluate the reliability of the methods used to discriminate between slightly different 
models, we introduced a synthetic $\mathrm{K}_{\mathrm{v}} 1.2$ test set, with shifted helices. We found limited discriminative power for this decoy set (Table 2B). Part of the reason for this is that none of the methods used was developed specifically for membrane proteins. Programs such as Verify3D take the local residue environment into account. Therefore, limited use of this method might be expected, because the environment for membrane proteins differs significantly. The membrane environment is predominantly hydrophobic with little possibility for hydrogen bonding and electrostatic interactions. This is also reflected in the differences in amino acid composition ${ }^{[42]}$ with different secondary-structure propensities between membrane environments and aqueous solution. ${ }^{[43]}$ Indeed, results obtained by Verify $3 \mathrm{D}$ are not able to discriminate between $\mathrm{K}_{\mathrm{v}} 1.2$ and the decoy set. We find that DFIRE and the local assessment method from Fasnacht et al. ${ }^{[30]}$ are able to discriminate between different alignments (Table 2 and Figure 3), whereas methods such as Procheck ( $\phi / \psi$ angles) are not suited for this task.

Law et al. ${ }^{[44]}$ found a good correlation between quality as assessed with static structure assessment and stability in MD simulations. Whether MD simulations are suitable to distinguish between correctly and incorrectly folded models, for example, via misalignment of certain segments is still an open question. If helices are incorrectly packed one might expect to see a greater degree of drift from the starting coordinates reflected in large RMSD values. ${ }^{[45-47]}$ Simulations have also proven helpful to distinguish between different Kir6.2 alignments, ${ }^{[44]}$ and we have previously shown that a distinction between clockwise and counterclockwise orientation in an L-type calcium channel model is possible. ${ }^{[48]}$ On the other hand, Law et al. ${ }^{[44]}$ report that it is not possible to distinguish between different subunit orientations in Twk- 5 channels. Clearly this issue is inconclusive. In hERG models, large drifts (i.e., repacking of helices) were observed only for three out of the seven models (Figure 4A). The simulations suggest that one can identify models that contain serious problems, such as model 2 , which scored poorly in most of the static assessments and also displays a rather high RMSD value. However, models 1 and 6, which are ranked among the best models, are difficult to distinguish using MD simulations. Simulations for these two models were extended to 60 (m1) and $100 \mathrm{~ns}$ (m6), with still no clear distinction between them (Figure $4 \mathrm{C}$ ). This suggests that although MD can identify poor model quality, low RMSD values do not automatically identify the correct fold.

Different RMSD values reflect packing differences between S5 and S6/P-helix. The exception is model 2 , which has a somewhat longer sequence (S5 intracellular), but it is unlikely the sole reason for the higher RMSD values observed for this model. At the end of the $10 \mathrm{~ns}$ simulation, no equilibrium is reached.

Significant differences in stability could not be detected in our $\mathrm{K}_{\mathrm{v}} 1.2$ decoy test sets, in which we mimicked incorrect packing between the outer M1 (corresponding to S5) and inner M2 (corresponding to S6) helices, by introducing artificial shifts in the outer helices (Figure 4B). This might be at least partially explained by the fact that $\mathrm{M} 1$ is rather symmetric, and changes in helix-helix packing upon shifts are relatively minor (Figure 4C).
In three out of the seven hERG models ( $\mathrm{m} 1, \mathrm{~m} 3$, and $\mathrm{m} 5$ ) a drastic decrease in cavity size was observed due to a collapse of all four aromatic F656 residues. This effect has been described previously for model $3 .^{[12]}$ The authors reported the unsuitability of the model for docking studies after 3.5 ns simulation, which is in agreement with our analysis. It is reasonable to assume that the orientation of the pore residues varies with the channel state (i.e., inactivated-activated-deactivated; for examples, see references [31-33]), and we cannot exclude that this "collapse" represents such a state. However, repeated collapses on a very short time scale (several nanoseconds) are at least suspicious, as although inactivation in hERG is fast, it occurs on the millisecond time scale. Additionally, MD simulations of a high-resolution crystal structure of the NaK cation channel in the open conformation, which also contains four pore-facing Phe residues, did not lead to similar narrow cavities (our unpublished observations). Our study suggests that there is a correlation between pore collapse (see Figure 5) and (mis)alignment.

Experimental validation of the S5 segments comes from a recent alanine scan by Ju et al. ${ }^{[1]}$ These data agree best with the alignment of model 6 and thus confirm this model as likely open conformation hERG structure (Figure 6). This alanine scan is least compatible with model 2, which was constantly ranked lowest with various programs.

Ju et al. ${ }^{[1]}$ show that mutations on the S5 helix are capable of strongly interfering with the energetics of inactivation and activation gating in hERG. This underscores the importance of modeling this section of hERG in addition to the cavity-forming helixes S6, P, and the selectivity filter, in order to study the molecular basis of hERG kinetics and its possible impact on drug binding, as inactivation plays an important role in high-affinity drug interactions.

Most residues on S5 experimentally shown to impact inactivation energetics were found to face $\mathrm{S} 6$ in our model. The effect of the mutant F557A is especially intriguing, because it decreases the barrier toward inactivation substantially. The phenyl ring of F557 directly faces the side chain of Y652 on S6 in our model. Recent experimental work by Klement et al., ${ }^{[32]}$ in which the effect of the hERG-like mutation $1470 \mathrm{Y}$ on the inactivation properties of Shaker (1470 is homologous to Y652 in hERG) was studied, shows that the mutation effects a considerable acceleration of the C-type component of inactivation. Klement et al. ${ }^{[32]}$ suggest that it is the rotameric state of the tyrosine side chain (cavity-pointing vs. cavity-lining) which is most crucially involved in the induction of this inactivated state. The close stacking interaction between Y652 and F557 observed in our model could serve as a basis to explain the strong effect of this S5 residue on inactivation, as mutation of F557 most probably influences the conformational state of $\mathrm{Y} 652$ in this environment.

Very recently Lees-Miller et al. ${ }^{[49]}$ reported interactions of segment S5 with the pore helix of hERG. The alignment in their paper corresponds to the alignment of model 1 described herein. This model scores second best in our overall analysis; however, it is in contradiction with experiments reported by Ju et al. ${ }^{[1]}$ Although the overall structure of model 1 was stable in 
MD simulations, the pore-facing F656 residues were found to collapse during MD simulations. In our model 1, the proposed hydrogen bonding network between $\mathrm{H} 562$ from S5 and T618 and $\mathrm{S} 621$ from the P-helix was not observed. We therefore rebuilt model 1 using the newest Modeller version (9v6), but again, in none of the 100 generated structures were hydrogen bonds between $\mathrm{H} 562$ and $\mathrm{T} 618$ and $\mathrm{S} 621$ present. To address the question if such a putative hydrogen bonding network would influence the stability of the inner pore, that is, prevent collapse of the F656 rings, we performed two independent MD simulations of $20 \mathrm{~ns}$ including distance restraints (force constants 10 and $100 \mathrm{~kJ} \mathrm{~mol}^{-1} \mathrm{~nm}^{-2}$ ) to enforce similar hydrogen bonds as suggested by Lees-Miller et al. ${ }^{[49]}$ In both simulations, only T618 was able to form hydrogen bonds with H562; however, S621 was found to be too far away $(\sim 6.4 \AA)$ from the histidine side chain. Furthermore, no influence on the stability of the inner cavity was observed. It is not clear if model 6 , as proposed by our work, can explain the mutational data on $\mathrm{H} 562$, because in this model residue $\mathrm{H} 562$ is orientated toward the voltage-sensing domain, which was not modeled.

Additionally, we performed a limited docking study on the well-studied high-affinity blockers (+)-cisapride, (S)-terfenadine, and MK-499. ${ }^{[10,25]}$ The outcome of our limited drug-receptor evaluation is, with the exception of model 2, in good agreement with results obtained by static assessment methods and MD simulations. Docking into model 6, which scored best with most assessment methods, yielded docking results that are in good agreement with alanine scan experiments. ${ }^{[10,25]} \mathrm{Re}-$ sults for model 6 are also partly in agreement with recent chimera studies by Imai et al. ${ }^{[33]}$ and Myokai et al., ${ }^{[50]}$ in which the nature of aromatic interactions for cisapride and terfenadine were studied. In agreement with Kamiya et al., ${ }^{[25]}$ hydrogen bonds to the selectivity filter residues are predicted.

No hydrogen bonds between the phenol side chain of Y652 and any of the three studied high-affinity drugs were predicted, which agrees with the study by Fernandez et al. ${ }^{[51]}$ However, docking into static models cannot explain the importance of V625 or G648, which influence the affinity of MK-499. ${ }^{[10]}$ It might be possible that these residues interact with blockers via an indirect mechanism.

Cisapride was docked into all seven models, but did not fit into the collapsed pores of models 1,3 , and 5 . In model 1 , which scored second best in our quality assessment, two additional drugs (terfenadine and MK-499) were docked with two different programs. However, none of the tested drugs could be accommodated in the narrow cavity. This is surprising because there is good evidence that certain drugs such as MK499 might be trapped in the closed channel pore and thus could possibly fit into a narrow cavity. Furthermore, docking studies, with blockers in the closed hERG channels have been published (for examples, see references $[10,13,15,34]$ ). This prompted us to compare the cavities of model 1 with a closed channel cavity. Surprisingly, the volume of the collapsed pore is smaller than the cavity of a closed channel pore (our unpublished observations). Therefore, we suggest a possible relationship between misalignment and pore collapse in MD simulations. In agreement with this hypothesis, the size of the inner cavity for model 6 does not change significantly over time, and no collapse of the pore (up to $100 \mathrm{~ns}$ ) emerges.

Surprisingly, model 2 performed quite well in docking analysis, despite the poor values obtained in quality assessments. It is likely that the results for model 2 are influenced by the adjustments of glycine residues in S5 and S6, leading to an artificially large inner cavity. ${ }^{[11]}$ Reasonable binding modes could be obtained for the best and the lowest scoring model, highlighting the limits of static docking methods to validate models. One major disadvantage of such methods is the limited possibility to address receptor dynamics, which will be an essential step toward understanding the promiscuity of hERG. The model of the hERG structure presented in this study provides a basis for addressing the relation between ligand affinity and hERG conformational dynamics, using methods that take the receptor dynamics explicitly into account. In particular, the model can help to study aspects of channel kinetics such as mechanisms inducing entry into inactivation and activation gating in greater detail. Dependence of high-affinity drug binding on conformational changes related to inactivation gating has been described before, ${ }^{[50,52]}$ and some members of the relatively newly discovered class of channel activators appear to work through inhibition of channel C-type inactivation, ${ }^{[53]}$ whereas others seem to prevent deactivation. ${ }^{[54 a]}$ The elucidation of these conformational changes also requires knowledge of the electrostatic properties of the entire pore region. A complete and reliable molecular model of the pore-forming part of hERG is a prerequisite to understand these mechanisms and their possible influence on the characteristic promiscuity of drug binding.

\section{Limitations}

The major aim of this study was the identification of the correct alignment for segment S5 in hERG. We did not focus on detailed refinement of the models, and although model 6 fits the experimental data for S5 well, we cannot rule out that further refinement might be necessary, for example, to study inactivation. Furthermore, structurally important segments such as the S5 turret helices and the voltage-sensing domains are missing. Moreover, the structural quality of the models presented herein is limited by the resolution of the $\mathrm{K}_{\mathrm{v}} \mathrm{AP}$ template (3.2 $\AA$ ). Nevertheless, we view the structural model presented here as an important step, as it represents the most plausible model of the hERG inner pore structure based on currently available data, and provides a necessary prerequisite to study the determinants of ligand binding to the hERG inner cavity.

\section{Conclusions}

It is critically important to use a combination of methods to assess the quality of homology models. ${ }^{[44]}$ Careful model evaluation is crucial, especially when target and template are distantly related (i.e. below the $30 \%$ identity threshold). With a combination of static assessment programs, MD simulations, and experimental validation, we identified the most likely alignment for hERG out of seven suggested possibilities. Our study clearly shows that a careful evaluation of model quality 
is able to distinguish between different alignments. Furthermore, we show that alignment errors, even in segments not directly involved in drug interactions, can severely influence the shape and size of the binding site. Using this combined ap proach, we propose a consensus model of the hERG potassium channel structure that can be used as a basis for structurebased ligand affinity predictions, to study structure-function relationships, and to inspire future experiments.

\section{Experimental Section}

\section{Model building}

We used Modeller $7 \mathrm{v} 7^{[55]}$ to generate homology models of the open conformation of the human ERG1 (accession number: Q12809) channel using the $K_{v} A P$ crystal structure (PDB ID: 1ORQ) and a refined model thereof $f^{56]}$ as templates. $K_{v} A P$ was preferred over $\mathrm{K}_{\mathrm{v}} 1.2$ because the latter channel contains a PXP motif in the inner $\mathrm{S} 6$ segments, which might change the shape of the pore. ${ }^{[51]}$ Furthermore, the sequence of $K_{v} A P$ is more similar to that of the hERG pore domain. Alignments 2-6 were extracted from published sources, ${ }^{[11-14,16]}$ and alignments for models 1 and 7 were added for completeness. Fourfold symmetry was imposed for modeling the tetrameric structures of the pore-forming domains including S5 segments, P-helices, re-entrant loops, and S6 segments. Models do not include the S5-P linkers and the voltage-sensing domains. Additionally, a synthetic test set containing the $\mathrm{K}_{\mathrm{v}} 1.2 / 2.1$ chimera pore domain (PDB ID: 2R9R) plus eight models with shifted S5 segments were built.

\section{Static quality assessment}

Verify3D, ${ }^{[57]}$ Procheck $\phi / \psi$ angle check, ${ }^{[58]}$ WHAT_CHECK Packing $2{ }^{[59]}$ Prosa2003, ${ }^{[60]}$ ProQres, ${ }^{[66]}$ DFIRE, ${ }^{[62]}$ ModFOLD, ${ }^{[63]}$ and a local quality assessment method developed by Fasnacht et al., ${ }^{[30]}$ were used to assess the quality of various models.

\section{Brief description of quality assessment methods}

Verify $3 D^{[57]}$ is a knowledge-based method that uses statistical potentials from real proteins and assesses how well a sequence fits its 3D structure by taking into account the residue environment combining secondary structure, solvent accessibility, and polarity. Procheck ${ }^{[58]}$ assesses the stereochemical parameters of a protein. The Ramachandran plot shows the $\phi / \psi$ torsion angles for all residues in the structure, defining different regions ranging from highly populated to very unusual or "forbidden" values. WHAT_CHECK Packing ${ }^{[59]}$ uses "fixed fragments" in a protein structure and checks the occurrence of all possible atom types in all possible positions around these fragments. Frequently occurring configurations are considered preferred. A summary score for each residue is calculated. Prosa2003 ${ }^{[60]}$ uses distance- and surface-dependent statistical potentials for $\mathrm{C}^{\alpha}$ atoms of all residues in the model. ProQres ${ }^{[6]]}$ is a structure-based method that analyzes atom-atom contacts, residue-residue contacts, solvent-accessible surfaces, and secondary structure. The DFIRE score is a statistical potential summed over all pairs of non-hydrogen atoms. As reference state, $\mathrm{DFIRE}^{[62]}$ uses a distance-scaled finite ideal gas. The ModFOLD method ${ }^{[63]}$ which is available as web sever, combines data from ModSSEA, ${ }^{[30]}$ MODCHECK, ${ }^{[64]}$ and ProQ $^{[65]}$ using a neural network to predict the accuracy of a model. The method developed by Fasnacht et al. ${ }^{[30]}$ uses a combination of different statistical potentials (DFIRE, contact and torsion potentials), and structural features making use of programs such as DSSP, ${ }^{[66]}$ psipred, ${ }^{[67]}$ and Verify3D, using a support vector machine to assess the local quality of a model.

\section{Molecular dynamics simulations}

MD simulations were performed with Gromacs v. 3.3. ${ }^{[68]}$ All hERG models, as well as crystal structures of $\mathrm{K}_{\mathrm{v}} \mathrm{AP}$ and $\mathrm{K}_{\mathrm{v}} 1.2$ plus synthetic models, were embedded in an equilibrated simulation box of 241 palmitoyloleoyl phosphatidylcholine (POPC) lipids. The channels were inserted into the membrane as described previously. ${ }^{[69]}$ $\mathrm{K}^{+}$ions were placed in the channel at $\mathrm{K}^{+}$sites $\mathrm{S} 0, \mathrm{~S} 2$, and $\mathrm{S} 4$, with waters placed at $\mathrm{S} 1$ and $\mathrm{S} 3$ of the selectivity filter. ${ }^{[70]} \mathrm{Cl}^{-}$ions were added randomly within the solvent to neutralize the system. Identical simulations with an ionic strength of $150 \mathrm{~mm}$ were also carried out. Lipid parameters were taken from Berger et al., ${ }^{[71]}$ and the OPLS-all-atom force field ${ }^{[72]}$ was used for the protein. The solvent was described by the TIP4P water model. ${ }^{[73]}$ Electrostatic interactions were calculated explicitly at a distance $<1 \mathrm{~nm}$, and longrange electrostatic interactions were calculated at every step by particle-mesh Ewald summation. ${ }^{[74]}$ Lennard-Jones interactions were calculated with a cutoff of $1 \mathrm{~nm}$. All bonds were constrained by using the LINCS algorithm, ${ }^{[75]}$ allowing for an integration time step of $2 \mathrm{fs}$. The simulation temperature was kept constant by weakly ( $\tau=0.1 \mathrm{ps)}$ coupling the lipids, protein, and solvent (water + counter-ions) separately to a temperature bath of $300 \mathrm{~K}$. The pressure was kept constant by weakly coupling the system to a pressure bath of 1 bar with semi-isotropic pressure coupling. Prior to simulations, 500 conjugate gradient energy-minimization steps were performed, followed by $2 \mathrm{~ns}$ of restrained MD in which the protein atoms were restrained with a force constant of $1000 \mathrm{~kJ} \mathrm{~mol}^{-1} \mathrm{~nm}^{-2}$ to their initial position. lons, lipids, and solvent were allowed to move freely during this $2 \mathrm{~ns}$ equilibration phase. The system was then subjected to $20 \mathrm{~ns}$ of unrestrained MD, during which coordinates were saved every 10 ps for analysis. Parts of models 2 and 7 started to unfold after several ns; therefore, simulations were stopped after $10 \mathrm{~ns}$. Simulations for models 1 and 6 were extended to 60 and $100 \mathrm{~ns}$, respectively. $\mathrm{p} K_{\mathrm{a}}$ values for all titratable amino acid side chains within the models were calculated using PROPKA. ${ }^{[76]}$ Residues at the $\mathrm{N}$ - and C-termini were considered as uncharged, as neither lie at the actual termini of the complete channel.

\section{Drug docking}

FlexX v. 3.0.2 $2^{[77]}$ and the GOLD evaluation v. 4.0.1 $1^{[78]}$ with standard parameters were used to analyze hERG interactions with (+)-cisapride, (S)-terfenadine, and MK-499. Drug coordinates were obtained from the PubChem structure database. ${ }^{[79]}$ The starting geometries of the drugs were optimized with the Hartree-Fock, 6$31 \mathrm{G}^{*}$ basis set, as implemented in Gaussian 03. ${ }^{[54 b]}$ Structures prior to MD simulations and minimized averaged structures (final 500 ps) were used as starting coordinates for docking. The highestscoring docking poses for each model were stored, and results of different hERG models were compared.

\section{Acknowledgements}

We thank Ulrike Gerischer for carefully reading the manuscript. This work was funded by TI Pharma project D2-101 (A.S. and 
U.Z.) and EC project HEALTH-F4-2007-201924, EDICT Consortium (S.J.W.).

Keywords: docking $\cdot$ hERG - model validation - molecular dynamics $\cdot$ molecular modeling

[1] P. Ju, G. Pages, R. P. Riek, P. C. Chen, A. M. Torres, P. S. Bansal, S. Kuyucak, P. W. Kuchel, J. I. Vandenberg, J. Biol. Chem. 2009, 284, 1000-1008.

[2] M. C. Sanguinetti, C. Jiang, M. E. Curran, M. T. Keating, Cell 1995, 81, 299-307.

[3] M. C. Trudeau, J. W. Warmke, B. Ganetzky, G. A. Robertson, Science 1995 269, $92-95$

[4] G. N. Tseng, J. Mol. Cell. Cardiol. 2001, 33, 835-849.

[5] J. I. Vandenberg, B. D. Walker, T. J. Campbell, Trends Pharmacol. Sci. 2001, 22, 240-246.

[6] A. Arcangeli, L. Bianchi, A. Becchetti, L. Faravelli, M. Colonnello, E. Mini, M. Olivotto, E. Wanke, J. Physiol. 1995, 489, 455-471.

[7] M. E. Curran, I. Splawski, K. W. Timothy, G. M. Vincent, E. D. Green, M. T. Keating, Cell 1995, 80, 795-803.

[8] R. Pearlstein, R. Vaz, D. Rampe, J. Med. Chem. 2003, 46, 2017-2022.

[9] C. Jamieson, E. M. Moir, Z. Rankovic, G. Wishart, J. Med. Chem. 2006, 49, 5029-5046.

[10] J. S. Mitcheson, J. Chen, M. Lin, C. Culberson, M. C. Sanguinetti, Proc Natl. Acad. Sci. USA 2000, 97, 12329-12333.

[11] R. Farid, T. Day, R. A. Friesner, R. A. Pearlstein, Bioorg. Med. Chem. 2006, $14,3160-3173$.

[12] M. Masetti, A. Cavalli, M. Recanatini, J. Comput. Chem. 2008, 29, $795-$ 808.

[13] P. J. Stansfeld, P. Gedeck, M. Gosling, B. Cox, J. S. Mitcheson, M. J. Sutcliffe, Proteins 2007, 68, 568-580.

[14] G. N. Tseng, K. D. Sonawane, Y. V. Korolkova, M. Zhang, J. Liu, E. V. Grishin, H. R. Guy, Biophys. J. 2007, 92, 3524-3540.

[15] R. Rajamani, B. A. Tounge, J. Li, C. H. Reynolds, Bioorg. Med. Chem. Lett. 2005, 15, 1737-1741.

[16] F. Österberg, J. Åqvist, FEBS Lett. 2005, 579, 2939-2944.

[17] R. A. Pearlstein, R. J. Vaz, J. Kang, X. L. Chen, M. Preobrazhenskaya, A. E. Shchekotikhin, A. M. Korolev, L. N. Lysenkova, O. V. Miroshnikova, J. Hendrix, D. Rampe, Bioorg. Med. Chem. Lett. 2003, 13, 1829-1835.

[18] C. Chothia, A. M. Lesk, EMBO J. 1986, 5, 823-826.

[19] C. Sander, R. Schneider, Proteins 1991, 9, 56-68.

[20] D. A. Doyle, J. M. Cabral, R. A. Pfuetzner, A. Kuo, J. M. Gulbis, S. L. Cohen, B. T. Chait, R. MacKinnon, Science 1998, 280, 69-77.

[21] Y. Jiang, A. Lee, J. Chen, M. Cadene, B. T. Chait, R. MacKinnon, Nature 2002, 417, 515-522.

[22] Y. Jiang, A. Lee, J. Chen, V. Ruta, M. Cadene, B. T. Chait, R. MacKinnon, Nature 2003, 423, 33-41.

[23] S. B. Long, E. B. Campbell, R. MacKinnon, Science 2005, 309, 897-903.

[24] S. B. Long, X. Tao, E. B. Campbell, R. MacKinnon, Nature 2007, 450, $376-$ 382.

[25] K. Kamiya, R. Niwa, M. Morishima, H. Honjo, M. C. Sanguinetti, J. Pharmacol. Sci. 2008, 108, $301-307$.

[26] G. M. Clayton, S. Altieri, L. Heginbotham, V. M. Unger, J. H. MoraisCabral, Proc. Natl. Acad. Sci. USA 2008, 105, $1511-1515$

[27] H. P. Larsson, F. Elinder, Neuron 2000, 27, 573-583.

[28] J. Liu, M. Zhang, M. Jiang, G. N. Tseng, J. Gen. Physiol. 2002, 120, $723-$ 737.

[29] K. Hayashi, M. Shimizu, H. Ino, M. Yamaguchi, H. Mabuchi, N. Hoshi, H. Higashida, Cardiovasc. Res. 2002, 54, 67-76.

[30] M. Fasnacht, J. Zhu, B. Honig, Protein Sci. 2007, 16, 1557-1568.

[31] J. Chen, G. Seebohm, M. C. Sanguinetti, Proc. Natl. Acad. Sci. USA 2002, 99, $12461-12466$

[32] G. Klement, J. Nilsson, P. Arnhem, F. Elinder, Biophys. J. 2008, 94, $3014-$ 3022

[33] Y. N. Imai, S. Ryu, S. Oiki, J. Med. Chem. 2009, 52, 1630-1638.

[34] J. Karczewski, J. Wang, S. A. Kane, L. Kiss, K. S. Koblan, J. C. Culberson, R. H. Spencer, Biochem. Pharmacol. 2009, 77, 1602-1611.

[35] Y. Shafrir, S. R. Durell, H. R. Guy, Biophys. J. 2008, 95, 3650-3662.

[36] T. Kuner, P. H. Seeburg, H. R. Guy, Trends Neurosci. 2003, 26, 27-32.
[37] S. K. Burley, G. A. Petsko, Science 1985, 229, $23-28$.

[38] S. Persson, J. A. Killian, G. Lindblom, Biophys. J. 1998, 75, 1365-1371.

[39] I. T. Arkin, A. T. Brunger, Biochim. Biophys. Acta 1998, 1429, 113-128.

[40] C. Domene, S. Vemparala, M. L. Klein, C. Vénien-Bryan, D. A. Doyle, Biophys. J. 2006, 90, L01-L03.

[41] P. Braun, G. von Heijne, Biochemistry 1999, 38, 9778-9782.

[42] Y. Liu, D. M. Engelman, M. Gerstein, Genome Biol. 2002, 3, reserach0054.1-0054.12.

[43] S. C. Li, C. M. Deber, Nat. Struct. Biol. 1994, 1, 368-373.

[44] R. J. Law, C. Capener, M. Baaden, P. J. Bond, J. Campbell, G. Patargias, Y. Arinaminpathy, M. S. Sansom, J. Mol. Graphics Modell. 2005, 24, 157 165.

[45] L. R. Forrest, M. S. Sansom, Curr. Opin. Struct. Biol. 2000, 10, 174-181.

[46] A. Ivetac, M. S. Sansom, Eur. Biophys. J. 2008, 37, 403-409.

[47] J. F. Taly, A. Marin, J. F. Gibrat, BMC Bioinf. 2008, 9, 6.

[48] A. Stary, Y. Shafrir, S. Hering, P. Wolschann, H. R. Guy, Channels 2008, 2, $210-215$.

[49] J. P. Lees-Miller, J. O. Subbotina, J. Guo, V. Yarov-Yarovoy, S. Y. Noskov, H. J. Duff, Biophys. J. 2009, 96, 3600-3610.

[50] T. Myokai, S. Ryu, H. Shimizu, S. Oiki, Mol. Pharmacol. 2008, 73, $1643-$ 1651.

[51] D. Fernandez, A. Ghanta, G. W. Kauffman, M. C. Sanguinetti, J. Biol. Chem. 2004, 279, 10120-10127.

[52] E. Ficker, W. Jarolimek, J. Kiehn, A. Baumann, A. M. Brown, Circ. Res. $1998,82,368-395$

[53] O. Casis, S. P. Olesen, M. C. Sanguinetti, Mol. Pharmacol. 2006, 69, 658 665.

[54] a) M. Perry, F. B. Sachse, M. C. Sanguinetti, Proc. Natl. Acad. Sci. USA 2007, 104, 13827-13832; b) M. J. Frisch, Gaussian 03 (Revision C.02) Gaussian Inc., Wallingford, CT (USA), 2004.

[55] M. A. Marti-Renom, A. Stuart, A. Fiser, R. Sánchez, F. Melo, A. Sali, Annu. Rev. Biophys. Biomol. Struct. 2000, 29, $291-325$.

[56] S. Y. Lee, A. Lee, J. Chen, R. MacKinnon, Proc. Natl. Acad. Sci. USA 2005 $102,15441-15446$

[57] J. U. Bowie, R. Lüthy, D. Eisenberg, Science 1991, 253, 164-170.

[58] R. A. Laskowski, M. W. MacArthur, D. S. Moss, J. M. Thornton, J. Appl. Crystallogr. 1993, 26, 283-291.

[59] R. W. Hooft, G. Vriend, C. Sander, E. E. Abola, Nature 1996, 381, 272 272.

[60] M. Wiederstein, M. J. Sippl, Nucleic Acids Res. 2007, 35, W407-W410.

[61] P. Larsson, B. Wallner, E. Lindahl, A. Elofsson, Protein Sci. 2008, 17, $990-$ 1002.

[62] H. Li, Y. Zhou, J. Bioinf. Comput. Biol. 2005, 3, 1151-1170.

[63] L. J. McGuffin, Bioinformatics 2008, 24, 586-587.

[64] C. S. Pettitt, L. J. McGuffin, D. T. Jones, Bioinformatics 2005, 21, 3509 3515.

[65] L. J. McGuffin, BMC Bioinf. 2007, 8, 345.

[66] W. Kabsch, C. Sander, Biopolymers 1983, 22, 2577- 2637.

[67] D. T. Jones, J. Mol. Biol. 1999, 292, 195-202.

[68] E. Lindahl, B. Hess, D. van der Spoel, J. Mol. Model. 2001, 7, 306-317.

[69] J. D. Faraldo-Gómez, G. R. Smith, M. S. Sansom, Eur. Biophys. J. 2002, 31, 217-227.

[70] J. Åqvist, V. Luzhkov, Nature 2000, 404, 881-884.

[71] O. Berger, O. Edholm, F. Jähnig, Biophys. J. 1997, 72, 2002-2013.

[72] W. L. Jorgensen, D. S. Maxwell, J. Tirado-Rives, J. Am. Chem. Soc. 1996 $118,11225-11236$.

[73] W. L. Jorgensen, J. Chandrasekhar, J. D. Madura, R. W. Impey, M. L. Klein, J. Chem. Phys. 1983, 79, 926-935.

[74] T. Darden, D. York, L. Pedersen, J. Chem. Phys. 1993, 98, 10089-10092.

[75] B. Hess, H. Bekker, H. J.C. Berendsen, J. G. E. M. Fraaije, J. Comput Chem. 1997, 18, 1463-1472.

[76] H. Li, A. D. Robertson, J. H. Jensen, Proteins 2005, 61, 704-721.

[77] M. Rarey, FlexX 3.0.2, BioSolvelT GmbH, St. Augustin (Germany) 2008.

[78] G. Jones P. Willett, R. C. Glen, J. Mol. Biol. 1995, 245, 43-53.

[79] PubChem: http://pubchem.ncbi.nlm.nih.gov (accessed January 7, 2010).

Received: November 10, 2009

Revised: December 23, 2009

Published online on January 26, 2010 